\title{
Developments in treating metastatic colorectal cancer: Recent international reports from ASCO 2007 and 2008
}

\author{
Michel Ducreux \\ Gastro-Intestinal Unit, Institut \\ Gustave-Roussy, Villejuif Cedex, \\ France; Department of Oncology, Paul \\ Brousse University Hospital, Villejuif, \\ France; Paris-Sud XI University, Le \\ Kremlin Bicêtre, France
}

\begin{abstract}
Introduction: Treatment for metastatic colorectal cancer (mCRC), employing various schedules, combinations, and regimens utilizing 5-fluorouracil (5-FU), irinotecan, oxaliplatin, capecitabine, bevacizumab, and cetuximab, currently achieves an overall survival that extends to approximately two years. Major questions regarding optimal management of $\mathrm{mCRC}$ await resolution.

Methods: A thorough review was conducted of all mCRC abstracts, posters, and other presentations at the 2007 meeting of the American Society of Clinical Oncology (ASCO). Information was analyzed in relationship to previously published research to determine the potential impact of new data on current and future mCRC management strategies and patient outcomes. Updated data presented at ASCO 2008 relevant to these findings was also analyzed.

Discussion: Ongoing challenges in mCRC treatment include defining the optimal role of targeted agents such as cetuximab and bevacizumab, elaborating the mechanisms underlying their toxicities, resolving the benefits of adjuvant and neoadjuvant chemotherapy in patients who are candidates for surgical resection, establishing whether there are substantive differences between sequential and combination chemotherapy regimens, and determining the safety and tolerability of chemotherapy in elderly subjects.

Conclusion: Recent reports presented at ASCO 2007 and 2008 indicate incremental improvements in care of patients with mCRC. Nevertheless, irinotecan, oxaliplatin, 5-FU, and to an increasing extent the targeted biologic agents bevacizumab and cetuximab continue unchallenged as first-line and later selections.
\end{abstract}

Keywords: chemotherapy, combination chemotherapy, irinotecan, bevacizumab, cetuximab

\section{Introduction}

The annual meeting of the American Society of Clinical Oncology (ASCO) provides members of the international oncology community with vital educational exposure to the newest developments in chemotherapy for metastatic colorectal cancer (mCRC). The most recent meeting, in June 2007, highlighted important presentations from a number of European investigators and their American counterparts, with practical implications for improving the standard of cancer treatment and for increasing our understanding of the clinical potential contained in a burgeoning array of chemotherapy combinations and surgical strategies. Presentations describing the capabilities of newer agents and treatment-associated toxicities of these and more familiar agents were also of great interest and are discussed here. (Note that this article does not attempt to present complete investigator findings but to highlight areas of compelling interest. In many instances, due to time and space constraints inherent in written abstracts and oral presentations, $P$ values, confidence intervals, and other statistical information are not available).

\section{EPOC}

In plenary session, the European Organisation for Research and Treatment of Cancer (EORTC) presented final results of the EPOC (Eloxatin Peri-Operative Chemotherapy) 
study, a randomized phase III evaluation of the benefit of perioperative oxaliplatin, leucovorin (LV), and 5-fluorouracil (5-FU) (FOLFOX4) chemotherapy. ${ }^{1}$ The primary objective of EPOC was to demonstrate an improvement in progressionfree survival (PFS) with an initial treatment strategy that incorporated perioperative FOLFOX4 chemotherapy plus surgery compared with surgery alone. A notable aspect of this study was its pragmatic approach to the timing of chemotherapy: by design, there was no attempt to establish the superiority of either pre-operative or post-operative timing of administration. ${ }^{2}$ Rather, the investigators accommodated their investigation to the unique benefits of chemotherapy associated with each phase. Preoperative administration presumably confers benefits by shrinking tumor burden, thereby increasing the likelihood of smaller resectable masses and allowing the chemoresponsiveness of the tumor to be assessed. Postoperative administration targets dormant malignant cells in the hepatic remnant and is essentially an iteration of chemotherapy for stage III disease, with its known attendant benefits. $^{2}$

In EPOC, over a four-year period, 364 patients were randomly assigned to perioperative FOLFOX4 (six cycles before and six cycles after surgery) or surgery alone. The primary endpoint was PFS, with a projected goal of increasing median PFS by $40 \%$ (equivalent to a hazard ratio [HR] of 0.71). Comparable proportions of patients in each treatment group could not undergo resection, based on pre-operative imaging. In the chemotherapy arm, 151 patients were resected after a median of six preoperative cycles, and of these patients, 115 (63\%) underwent postoperative chemotherapy (also given for a median of six cycles). Perioperative chemotherapy improved PFS in patients with resectable hepatic lesions, and PFS improved even more in patients whose metastases were successfully resected, ${ }^{2}$ achieving a 9.2\% absolute difference in PFS at three years. ${ }^{1}$ Perioperative chemotherapy was safe, raising the possibility that this regimen warrants consideration as a new treatment standard. ${ }^{2}$ However, both the amount of accrual time and the high number of participating centers, with likely differences in liver surgery experience among the numerous teams, are weaknesses of this study. Furthermore, it should be emphasized that neoadjuvant chemotherapy before liver surgery is not completely safe, because in this study, it clearly increased the percentage of biliary fistulae (7.5\% versus $2.9 \%)$.

Results of the EPOC study are certain to elicit vigorous discussion, particularly as updated efficacy results of the MOSAIC (Multicenter International Study of Oxaliplatin/ 5FU-LV in the Adjuvant Treatment of Colon Cancer) study were also presented at the meeting. The MOSAIC study evaluated classical post-operative adjuvant therapy with FOLFOX4 in 2246 patients with completely resected stage II or III CRC and no prior chemotherapy. ${ }^{3}$ While MOSAIC was initially designed to evaluate the effects of FOLFOX4 on three-year disease-free survival (DFS), updated results for overall survival (OS) at longer intervals showed that FOLFOX4 achieved DFS benefits that were maintained at 3,5 , and 6 years, with no increase in the rate of secondary cancers. $^{3,4}$

Findings from studies such as EPOC and MOSAIC suggest a need for future trials that attempt a definitive comparison between neoadjuvant and adjuvant chemotherapy. Ongoing investigations comparing perioperative FOLFOX in combination with targeted biologic agents (cetuximab and bevacizumab; EORTC Trial 40051) were discussed by the EORTC investigators. $^{2}$

\section{CRYSTAL, EPIC, and NCIC CTG CO.I 7}

Cetuximab, a monoclonal antibody that specifically targets epidermal growth factor receptor (EGFR), was the focus of presentations reporting on three phase III clinical trials (CRYSTAL, EPIC, and CO.17). Both the CRYSTAL (Cetuximab combined with iRinotecan in first-line therapY for metaSTatic colorectaL cancer) and EPIC (European Prospective Investigation of Cancer) trials demonstrated that the addition of cetuximab to a standard irinotecan-containing regimen was associated with significant improvements on a number of outcome measures, including OS, PFS, tumor shrinkage, and patient quality of life.

CRYSTAL investigated the effectiveness of cetuximab in combination with standard irinotecan plus infusional 5-FU/LV (FOLFIRI) compared with FOLFIRI alone for firstline treatment of patients with metastatic, EGFR-expressing colorectal cancer. ${ }^{5}$ Patients were randomly assigned to cetuximab (400 mg/m² initial dose, then $250 \mathrm{mg} / \mathrm{m}^{2} /$ week) plus bi-weekly FOLFIRI (irinotecan $180 \mathrm{mg} / \mathrm{m}^{2}, \mathrm{LV} 400 \mathrm{mg} / \mathrm{m}^{2}$, 5 -FU bolus $400 \mathrm{mg} / \mathrm{m}^{2}, 5-\mathrm{FU}$ infusion $2400 \mathrm{mg} / \mathrm{m}^{2}$ over 46 hours) or FOLFIRI alone. The primary endpoint was PFS; secondary endpoints included overall response rate, disease control rates, OS, quality of life, and safety. The CRYSTAL trial met its primary objective, demonstrating that the addition of cetuximab to FOLFIRI for first-line treatment of EGFR-positive mCRC significantly increased PFS, with a $15 \%$ risk reduction for progression in patients treated with the combination. ${ }^{5}$ In addition, FOLFIRI plus cetuximab was associated with a significantly higher response 
rate $(46.9 \%$ versus $38.7 \%, \mathrm{P}=0.0038) .{ }^{6}$ In subsequent analysis, the investigators demonstrated that patients with initially unresectable disease who received FOLFIRI plus cetuximab had threefold higher radical $\left(\mathrm{R}_{0}\right)$ resection rates in comparison with patients receiving FOLFIRI alone, and patients with liver metastases as the only metastatic site had longer PFS than the whole population. ${ }^{7}$ Neutropenia was similar in both groups $(26.7 \%$ versus $23.3 \%$ ). Grade $3 / 4$ diarrhea occurred more frequently with the combination (15.2\% versus $10.5 \%$, respectively). Skin toxicity occurred more frequently with cetuximab treatment but was in the generally expected range $(18.7 \%$ versus $0.2 \%) .{ }^{5}$ Findings from CRYSTAL suggest that combination treatment with FOLFIRI plus cetuximab expands the list of triplet combinations that may be considered for first-line therapy. ${ }^{7}$

Data on the impact of KRAS mutation on the results of this trial were eagerly anticipated, as it has been suggested that patients with mutated KRAS tumors do not benefit from treatment with anti-EGFR inhibitors. ${ }^{8,9}$ Researchers analyzed archived tumor material from 540 KRAS-evaluable patients in the trial; these 540 were representative of the overall ITT population. ${ }^{10}$ Patients with wild-type KRAS who received cetuximab demonstrated significantly greater PFS (P = 0.0167; HR, 0.68 [95\% CI, 0.051-0.934]) and best overall response $(59.3 \%$ for patients receiving cetuximab + FOLFIRI versus $43.2 \%$ for those receiving FOLFIRI alone). However, patients with mutant-form KRAS did not demonstrate benefit from cetuximab treatment in terms of either PFS (P = 0.75; HR, 1.07 [95\% CI, 0.71-1.61]) or best overall response $(\mathrm{P}=0.46)$.

The EPIC study showed that the combination of cetuximab and irinotecan administered to patients with refractory $\mathrm{mCRC}$ as second-line therapy following oxaliplatin failure significantly improved PFS and provided a superior quality of life versus irinotecan alone. ${ }^{11,12}$ In this phase III clinical trial, pretreated patients were randomly assigned to receive either cetuximab $400 \mathrm{mg} / \mathrm{m}^{2}$ followed by $250 \mathrm{mg} / \mathrm{m}^{2}$ weekly and irinotecan $350 \mathrm{mg} / \mathrm{m}^{2}$ every three weeks $(\mathrm{n}=648)$ or irinotecan alone $(n=650)$. Although no difference in OS was noted between treatment arms, PFS and response rate were significantly better with the addition of cetuximab, and the combination of cetuximab and irinotecan resulted in better quality of life when compared with cytotoxic therapy alone, with less deterioration in global health status scores and symptom scores (pain, nausea, insomnia). ${ }^{12}$ The most interesting result of this study seems to be the better response rate in second-line treatment when cetuximab was added to irinotecan: $16 \%$ of patients receiving cetuximab plus irinotecan demonstrated a partial response, versus $4 \%$ receiving irinotecan alone $(\mathrm{P}<0.0001)$. This finding is of particular interest for patients with potentially resectable liver metastases and those who have failed first-line treatment with oxaliplatin-based chemotherapy.

In CO.17, a phase III randomized study of cetuximab and best supportive care (BSC) compared with BSC alone in patients with pre-treated EGFR-positive mCRC, conducted by the National Cancer Institute of Canada Clinical Trials Group in collaboration with the Australasian Gastro-Intestinal Trials Group, cetuximab administered as single-agent therapy to a patient population previously refractory to treatment was associated with significantly improved OS and provided a superior quality of life, in comparison with BSC alone. ${ }^{13}$ Median OS was 6.1 months with cetuximab plus BSC, versus 4.6 months for $\mathrm{BSC}$ alone $(\mathrm{P}=0.0046) .{ }^{14}$ Cetuximab is commonly used in combination with irinotecan among patients with refractory disease; thus the results of this study will not change standard practice. However, for patients with poor tolerance to irinotecan, this study showed that cetuximab is active alone.

\section{OPUS}

The comparative efficacy of FOLFOX4 plus cetuximab versus FOLFOX4 alone for initial treatment of previously untreated, unresectable mCRC was the focus of the OPUS (OxaliPlatin and cetUximab in firSt-line treatment of MCRC) trial. ${ }^{15}$ The primary outcome measure was overall response rate, defined as a decrease in tumor volume by $50 \%$ or more. Response rates favored FOLFOX plus cetuximab (45.6\% versus $35.7 \%$ ). ${ }^{15}$ Cetuximab administration was associated with a high rate of dermatologic toxicity (14.1\%) and infusion reactions; the latter, while noted in both treatment arms, were approximately twice as prevalent in the cetuximab group (4.1\% versus $1.8 \%){ }^{16}$ The results of this study are less favorable than those reported in an earlier, phase II trial of the combination of FOLFOX and cetuximab, in which the confirmed best overall response rate was $72 \%$ and the combination of cetuximab and FOLFOX-4 was generally well tolerated, with no evidence that cetuximab increased the frequency or severity of the characteristic toxicities associated with FOLFOX. ${ }^{17}$

In the OPUS trial, as in the CRYSTAL trial, KRAS status demonstrated a significant influence on efficacy. ${ }^{18}$ Investigators analyzed tissue from 233 patients, who were representative of the entire ITT population of the study. Patients with wild-type KRAS showed significantly greater PFS when treated with cetuximab + FOLFOX versus FOLFOX only 
(7.7 versus 7.2 months, respectively; HR, 0.57; $\mathrm{P}=0.02$ ); patients with KRAS mutation showed no significant benefit with the addition of cetuximab (5.5 versus 8.6 months; HR, $1.83 ; \mathrm{P}=0.02)$. Patients with wild-type KRAS also showed significantly greater response rates with the addition of cetuximab to FOLFOX (61\% versus $37 \%$; P = 0.01), while patients with KRAS mutation showed no significant benefit (33\% versus $49 \%$; $\mathrm{P}=0.11$ ).

\section{Cetuximab hypersensitivity}

Hypersensitivity reactions caused by cetuximab are a common, uncomfortable, and potentially unacceptable adverse effect. Intriguing data presented at ASCO 2007 suggest that these reactions may be mediated in many cases by pre-existing cetuximab-specific immunoglobulin $\mathrm{E}$ (IgE) antibodies (C-IgE). Investigators evaluated pre-treatment serum specimens from cetuximab-naïve mCRC patients and healthy volunteers. ${ }^{19}$ Of 71 cetuximab-treated subjects, $21(29.6 \%)$ experienced severe hypersensitivity, based on retrospective review of the medical record. In 15 of these patients, pretreatment sera were positive for $\mathrm{C}-\mathrm{IgE}$, and all 15 discontinued therapy. The remaining six patients from this group had C-IgE-negative sera; four were rechallenged uneventfully, while two received no further exposure. Analyses of sera from healthy volunteers revealed that 15 of $69(21.7 \%)$ were C-IgE-positive. These data strongly suggest that $\mathrm{C}-\mathrm{IgE}$ antibodies are present prior to treatment in a relatively high proportion of cetuximab-naïve subjects and are likely to be highly predictive of severe hypersensitivity reactions during cetuximab infusion. ${ }^{19}$

\section{BICC-C}

Updated efficacy results were reported at $\mathrm{ASCO}^{20}$ and subsequently published in Journal of Clinical Oncology ${ }^{21}$ for patients enrolled in the phase III randomized BICC-C study ( $n=430$ in period $1 ; n=117$ in period 2 , following a protocol amendment adding bevacizumab to the FOLFIRI and irinotecan plus bolus 5-FU/LV [mIFL] study arms). In this trial, initial treatment of $\mathrm{mCRC}$ with FOLFIRI or FOLFIRI plus bevacizumab was superior to a range of comparator regimens. Results presented at ASCO 2007 included, in period 1, median PFS of 7.6 months for patients receiving FOLFIRI, 5.9 months for mIFL ( $\mathrm{P}=0.004$ for the comparison with FOLFIRI), and 5.8 months for capecitabine and irinotecan (CapeIRI) $(\mathrm{P}=0.015)$. Median OS was 23.1 months among patients receiving FOLFIRI (with $75 \%$ of subjects surviving for at least one year), in comparison with $65 \%$ for mIFL and $66 \%$ for CapeIRI. In period 2, median PFS was 11.2 months for FOLFIRI plus bevacizumab and 8.3 months for mIFL plus bevacizumab $(\mathrm{P}=0.28) .{ }^{20}$ Median OS for patients receiving FOLFIRI plus bevacizumab had not been reached at the time of ASCO reporting; however, published results ${ }^{21}$ showed median OS of 28.0 months for patients receiving FOLFIRI plus bevacizumab, compared with 19.2 months for mIFL plus bevacizumab ( $\mathrm{P}=0.037$; HR for death, $1.79 ; 95 \%$ confidence interval [CI], 1.12-2.88). The 1-year survival rate was $87 \%$ for FOLFIRI plus bevacizumab, compared with $61 \%$ for mIFL plus bevacizumab. Grade 3 adverse events reported in period 2 included nausea $(5.1 \%)$, diarrhea $(11.9 \%)$, neutropenia $(28.8 \%)$, and febrile neutropenia $(1.7 \%)$. The median survival observed in this study is the best ever found in a trial of chemotherapy for metastatic colorectal cancer. With this combination, it now seems possible to break through the wall of 24-month survival that was previously considered very solid.

\section{CAIRO: Comparing the efficacy of sequential versus combination chemotherapy}

Median OS in mCRC improves with exposure to 5-FU/LV, irinotecan, and oxaliplatin - highly active cytotoxic drugs comprising the foundation of contemporary chemotherapy. ${ }^{22}$ No comparative data exist to determine whether exposure to sequential or combined use of these medications is preferred; similarly, the role of salvage treatments following failure of initial treatment has not been adequately studied in a prospective fashion. The Dutch Colorectal Cancer Group presented results at ASCO from the CAIRO (CApecitabine, IRinotecan, Oxaliplatin) study, the first phase III trial to prospectively evaluate sequential versus combination therapy in advanced CRC when all three cytotoxic drugs with known efficacy are made available ${ }^{23}$ (subsequently published in Lancet)..$^{24}$

The CAIRO investigators randomly assigned 820 previously untreated patients to sequential treatment with firstline capecitabine, second-line irinotecan, and third-line capecitabine plus oxaliplatin (arm A) or to combination chemotherapy with first-line CapIRI and second-line capecitabine and oxaliplatin (CapOx) (arm B). The dose of capecitabine was $1250 \mathrm{mg} / \mathrm{m}^{2}$ (sequential) or $1000 \mathrm{mg} / \mathrm{m}^{2}$ (combination) twice daily on days 1 through 14 , irinotecan $350 \mathrm{mg} / \mathrm{m}^{2}$ (sequential) or $250 \mathrm{mg} / \mathrm{m}^{2}$ (combination), and oxaliplatin $130 \mathrm{mg} / \mathrm{m}^{2}$. All cycles were administered every three weeks, with responses assessed every third cycle. ${ }^{24}$ Median follow-up was 32 months. ${ }^{24}$ 
Median OS, the primary endpoint, was 16.3 months with sequential therapy (95\% CI, 14.3-18.1), which was not significantly different from the 17.4 months achieved with combination therapy (95\% CI, 15.2-19.2; log rank $\mathrm{P}=0.3281) .{ }^{24}$ One-year survival rates were essentially identical (64\%, sequential; 67\%, combination). Overall grade $3 / 4$ toxicity across all lines did not differ significantly except for grade 3 hand-foot syndrome (13\% in arm A and $7 \%$ in arm B; $\mathrm{P}=0.004)$. Specific grade $3 / 4$ toxicities that were significantly more frequent with combination treatment were diarrhea $(\mathrm{P}<0.0001)$, vomiting $(\mathrm{P}=0.0002)$, nausea $(\mathrm{P}=0.004)$, and neutropenia, including febrile neutropenia $(\mathrm{P}=0.0001)$. Grade 3 hand-foot syndrome occurred significantly more often in conjunction with sequential chemotherapy $(\mathrm{P}=0.002)$. All-cause 60 -day mortality was $3.0 \%(\mathrm{n}=12)$ in arm A and $4.5 \%(\mathrm{n}=18)$ in arm B. The CAIRO findings suggest that while combination therapy does not significantly improve OS compared with sequential therapy, both treatment strategies are valid options for patients with advanced disease, given the high individual activity of the constituent cytotoxic agents.

The CAIRO and Fluorouracil, Oxaliplatin, and CPT11 (irinotecan) - Use and Sequencing (FOCUS) trials clearly demonstrate that it is possible to give first-line monotherapy to selected patients. In the FOCUS study, among 2135 treatment-naïve patients with advanced CRC, initial single-agent treatment upgraded to combination upon progression was noninferior to first-line combination treatment, with median survival of 13.9 months. ${ }^{25}$ However, only the combination of fluorouracil and irinotecan satisfied the statistical test for superiority, achieving median survival of 16.7 months $(\mathrm{P}=0.01)$. These concordant trials suggest that sequential treatment is an alternative option for discussion with patients that should be considered, especially for those with definitely nonresectable disease (ie, a combination of peritoneal carcinomatosis and liver metastases).

\section{BRiTE}

The BRiTE (Bevacizumab Regimens: Investigation of Treatment Effects and Safety) observational study reported on the utility of continuing first-line bevacizumab in salvage therapy for tumor progression. ${ }^{26}$ Using observational data from a registry encompassing nearly 2000 patients with $\mathrm{mCRC}$ receiving bevacizumab and first-line chemotherapy, the investigators assessed the effects on PFS and OS of continuing bevacizumab beyond progression (BBP), correcting for a range of pre- and posttreatment patient factors. First progression was documented in 1369 patients, occurring at a median follow-up of 17.5 months. In patients with progression, $65.2 \%$ received second-line chemotherapy with an approved agent, 34.8\% received cetuximab, and 53.8\% received BBP. While no chemotherapy sequence or combination predominated, multivariate analysis suggested that BBP or administration of any second-line chemotherapy was significantly associated with increased OS $(\mathrm{P}<0.0001) .{ }^{26}$ As with the CAIRO study, findings from the BRiTE registry emphasize that improved survival corresponds with exposure to all active agents.

\section{Chemotherapy in elderly patients}

The pervasive underrepresentation of elderly patients in clinical trials was the impetus for a phase II study of twicemonthly FOLFIRI administered to $\mathrm{mCRC}$ patients more than 70 years of age who were in otherwise good general condition. ${ }^{27}$ This analysis reported satisfactory efficacy and tolerability findings in 40 chemotherapy-naïve patients (median age, 77.3 years; range, 70-84.7 years) with performance status of 0 to 1 . Eleven patients were more than 80 years of age. Thirty-four patients $(85 \%)$ presented with one or more concomitant chronic conditions, and 23 patients $(58 \%)$ had at least three comorbid diseases. Patients received FOLFIRI every two weeks. In an intent-to-treat analysis, the objective response rate was 32.5\% (95\% CI, 18.6\%-49.1\%), including two complete responses and 11 partial responses. Median time to progression was 8.0 months, median OS was 14.2 months, and specific OS time was 18.6 months. Main toxicities were grade $3 / 4$ diarrhea $(n=13 ; 32.5 \%)$, asthenia $(n=6,15 \%)$, nausea $(n=3,7.5 \%)$, and neutropenia $(n=3,7.5 \%)$. One death was attributable to grade 4 diarrhea. Mean quality of life score, assessed using the Spitzer visual uniscale, increased from 75 at baseline to 87 at cycle $12(\mathrm{P}=0.005) .{ }^{27}$

Another presentation focusing on elderly patients was an updated meta-analysis of randomized trials evaluating 5-FU/LV with or without irinotecan in which, using original source data, the investigators compared the efficacy and toxicity of such treatment in older ( $\geq 70$ years) and younger patients $\left(<70\right.$ years) with $\mathrm{mCRC}^{28}$

A total of 2691 patient records were evaluated and clearly indicated that elderly patients enrolled in phase III trials derive benefits from irinotecan-containing chemotherapy comparable to those of younger patients, without excess risk of toxicity. Older and younger subjects demonstrated significantly improved response rates and PFS with irinotecan plus 5-FU/LV compared with 5-FU/LV alone. Younger patients had significantly longer OS with irinotecan plus 5-FU/LV, while older patients demonstrated a trend to longer OS with irinotecan plus 5-FU/LV. There were no 
significant differences regarding irinotecan plus 5-FU/LV toxicity between older and younger patients. ${ }^{28}$ These two trials clearly show that it is possible to administer aggressive chemotherapy to elderly patients. The suitability of these patients for chemotherapy has to be cautiously determined, but after this first step, there is no reason to decrease the intensity of chemotherapy in elderly patients.

\section{Conclusion}

Presentations at ASCO 2007 affirm that the steady accumulation of evidence continues upon which to base pharmacotherapy for metastatic colorectal cancer. While no report from this recent assembly, except the EPOC study, can be said to have immediate implications for changing clinical practices, it can also be said that the current foundations of care - irinotecan, oxaliplatin, 5-FU, and to an increasing extent the targeted biologic agents bevacizumab and cetuximab - continue unchallenged as first-line and later therapy selections. It now seems possible to give cetuximab in the first line to patients with $\mathrm{mCRC}$. However, knowing the results of biologic determinants of efficacy such as KRAS is necessary to choose the best population to treat with this agent. This ASCO meeting did not add information for the administration or efficacy of bevacizumab, except that the BICC-C trial gave us the opportunity to see the best median overall survival results ever encountered in nonselected patients with metastatic colorectal cancer.

\section{Disclosure}

Editorial assistance for this manuscript was provided by Adelphi, Inc., and funded by Pfizer, Inc.

\section{References}

1. Nordlinger B, Sorbye H, Collette L, et al. Final results of the EORTC Intergroup randomized phase III study 40983 [EPOC] evaluating the benefit of peri-operative FOLFOX4 chemotherapy for patients with potentially respectable colorectal cancer liver metastases. Proc Am Soc Clin Oncol. 2007;25:LBA5.

2. Nordlinger B. Peri-operative FOLFOX4 chemotherapy and surgery for resectable liver metastases from colorectal cancer: Final efficacy results of the EORTC Intergroup phase III study 40983 [slide presentation]. Presented at Proceedings of the American Society of Clinical Oncology 43rd Annual Meeting; June 1-5, 2007; Chicago, IL.

3. De Gramont A. Oxaliplatin/5FU/LV in adjuvant colon cancer: Updated efficacy results of the MOSAIC trial, including survival, with a median follow-up of 6 years [slide presentation]. Presented at Proceedings of the American Society of Clinical Oncology 43rd Annual Meeting; June 1-5, 2007; Chicago, IL, USA.

4. De Gramont A, Boni C, Navarro M, et al. Oxaliplatin/5FU/LV in adjuvant colon cancer: Updated efficacy results of the MOSAIC trial, including survival, with a median follow-up of six years. Proc Am Soc Clin Oncol. 2007;25:4007.

5. Van Cutsem E, Nowacki M, Lang I, et al. Randomized phase III study of irinotecan and 5-FU/FA with or without cetuximab in the first-line treatment of patients with metastatic colorectal cancer (mCRC): The CRYSTAL trial. Proc Am Soc Clin Oncol. 2007;25:4000.
6. Benson AB. Best of ASCO: gastrointestinal (colorectal) cancer [slide presentation]. Presented at Proceedings of the American Society of Clinical Oncology 43rd Annual Meeting; June 1-5, 2007; Chicago, IL, USA.

7. Grem J. 2007 Best of ASCO gastrointestinal (colorectal) cancer [slide presentation on the Internet]. 2007. Alexandria (VA): American Society of Clinical Oncology. [Cited on April 30, 2008]. Available from: http://asco.org/portal/site/ASCO/menuitem.64cfbd0f85cb37b2eda2be 0aee37a01d/?vgnextoid=09f8201eb61a7010VgnVCM100000ed730ad 1RCRD\&vmview=vm_meeting_tracks_view\&index=y\&confID=51.

8. Lievre A, Bachet JB, Boige V, et al. KRAS mutations as an independent prognostic factor in patients with advanced colorectal cancer treated with cetuximab. J Clin Oncol. 2008;26:374-379.

9. Lievre A, Bachet JB, Le Corre D, et al. KRAS mutation status is predictive of response to cetuximab therapy in colorectal cancer. Cancer Res. 2006;66:3992-3995.

10. Van Cutsem E, Lang I, D'haens G, et al. KRAS status and efficacy in the first-line treatment of patients with metastatic colorectal cancer (mCRC) treated with FOLFIRI with or without cetuximab: The CRYSTAL experience. Proc Am Soc Clin Oncol. 2008;26:2.

11. Eng C. Impact on quality of life of adding cetuximab to irinotecan in patients who have failed prior oxaliplatin-based therapy: Results from the EPIC trial [slide presentation]. Presented at Proceedings of the American Society of Clinical Oncology 43rd Annual Meeting; June 1-5, 2007; Chicago, IL, USA.

12. Eng C, Maurel J, Scheithauer W, et al. Impact on quality of life of adding cetuximab to irinotecan in patients who have failed prior oxaliplatin-based therapy: The EPIC trial. Proc Am Soc Clin Oncol. 2007;25:4003.

13. Au H, Karapetis C, Jonker D, et al. Quality of life in patients with advanced colorectal cancer treated with cetuximab: Results of the NCIC CTG and AGITG CO.17 trial. Proc Am Soc Clin Oncol. 2007;25:4002.

14. Au H. Quality of life in patients with advanced colorectal cancer treated with cetuximab: Results of the NCIC CTG and AGITG CO.17 trial [slide presentation]. Presented at Proceedings of the American Society of Clinical Oncology 43rd Annual Meeting; June 1-5, 2007; Chicago, IL, USA.

15. Bokemeyer C, Bondarenko I, Makhson A, et al. Cetuximab plus 5-FU/FA/oxaliplatin (FOLFOX-4) versus FOLFOX-4 in the first-line treatment of metastatic colorectal cancer (mCRC): OPUS, a randomized phase II study. Proc Am Soc Clin Oncol. 2007;25:4035.

16. Bokemeyer C. Cetuximab plus 5-FU/FA/oxaliplatin (FOLFOX-4) in the first-line treatment of mCRC: OPUS, a phase II study [slide presentation]. Presented at Proceedings of the American Society of Clinical Oncology 43rd Annual Meeting; June 1-5, 2007; Chicago, IL, USA.

17. Tabernero J, Van Cutsem E, Díaz-Rubio E, et al. Phase II trial of cetuximab in combination with fluorouracil, leucovorin, and oxaliplatin in the first-line treatment of metastatic colorectal cancer. J Clin Oncol. 2007;25:5225-5232.

18. Bokemeyer C, Bondarenko I, Hartmann JT, et al. KRAS status and efficacy of first-line treatment of patients with metastatic colorectal cancer (mCRC) with FOLFOX with or without cetuximab: The OPUS experience. Proc Am Soc Clin Oncol. 2008;26:4000.

19. Chung $\mathrm{CH}$, Chan E, Berlin J, et al. Cetuximab-related hypersensitivity reactions associated with pre-existing cetuximab-specific IgE antibody. Proc Am Soc Clin Oncol. 2007;25:9097.

20. Fuchs C, Marshall J, Mitchell E, et al. Updated results of BICC-C study comparing first-line irinotecan/fluoropyrmidine combinations with or without celecoxib in mCRC: Updated efficacy data. Proc Am Soc Clin Oncol. 2007;25:4027.

21. Fuchs CS, Marshall J, Mitchell E, et al. Randomized, controlled trial of irinotecan plus infusional, bolus, or oral fluoropyrimidines in firstline treatment of metastatic colorectal cancer: results from the BICC-C study. J Clin Oncol. 2007;25:4779-4786.

22. Grothey A, Sargent D, Goldberg RM, Schmoll HJ. Survival of patients with advanced colorectal cancer improves with the availability of fluorouracil-leucovorin, irinotecan, and oxaliplatin in the course of treatment. J Clin Oncol. 2004;22:1209-1214. 
23. Punt CJ, Koopman M, Douma J, et al. Sequential compared to combination chemotherapy with capecitabine, irinotecan, and oxaliplatin in advanced colorectal cancer (ACC): a Dutch Colorectal Cancer Group (DCCG) phase III study. Proc Am Soc Clin Oncol. 2007;25:4012.

24. Koopman M, Antonini NF, Douma J, et al. Sequential versus combination chemotherapy with capecitabine, irinotecan, and oxaliplatin in advanced colorectal cancer (CAIRO): a phase III randomised controlled trial. Lancet. 2007;370:135-142.

25. Seymour MT, Maughan TS, Ledermann JA, et al; FOCUS Trial Investigators; National Cancer Research Institute Colorectal Clinical Studies Group. Different strategies of sequential and combination chemotherapy for patients with poor prognosis advanced colorectal cancer (MRC FOCUS): a randomised controlled trial. Lancet 2007;370:143-152. Erratum in: Lancet. 2007;370:566.
26. Grothey A, Sugrue M, Hedrick E, et al. Association between exposure to bevacizumab (BV) beyond first progression (BBP) and overall survival (OS) in patients (pts) with metastatic colorectal cancer (mCRC): Results from a large observational study (BRiTE). Proc Am Soc Clin Oncol. 2007;25:4036.

27. Francois E, Chamorey E, Berdah J, et al. Phase II assessing irinotecan (CPT11) plus 5-fluorouracil (5FU) and leucovorin (FOLFIRI regimen) as first-line chemotherapy for elderly patients with metastatic colorectal cancer. Proc Am Soc Clin Oncol. 2007;25:314.

28. Folprecht G, Seymour MT, Saltz L, et al. Irinotecan/5-FU/FA (I-FU) or 5-FU/FA (FU) first-line therapy in older and younger patients with metastatic colorectal cancer: Combined analysis of 2,691 patients in randomized controlled trials. Proc Am Soc Clin Oncol. 2007;25:4071 
